\title{
Microneedle Based ECG - Glucose Painless MEMS Sensor with Analog Front End for Portable Devices
}

\author{
Miguel Lima Teixeira ${ }^{1,2}$, Camilo Velez ${ }^{2}$, Dian Li $^{3}$ and João Goes ${ }^{1}$, \\ ${ }^{1}$ Centre for Technologies and Systems (CTS) - UNINOVA, Dept. of Electrical Engineering \\ (DEE), Faculty of Sciences and Technology (FCT NOVA), NOVA University of Lisbon,2829- \\ 516, Monte da Caparica, Portugal \\ mdl.teixeira@campus.fct.unl.pt \\ ${ }^{2}$ Electrical and Computer Engineering Dept. (ECE), University of Florida, \\ 32611, Gainesville, Florida, USA \\ ${ }^{3}$ Schepens Eye Research Institute, Massachusetts Eye and Ear, \\ 02114, Boston, Massachusetts, USA
}

\begin{abstract}
A portable microelectromechanical system (MEMS) for mobile phones, or other portable devices, that measures body electrical signals, as well as, extracts transdermal biological fluid for invivo analysis is proposed. This system integrates two sensing methods: three points finger electrocardiography (ECG) and glucose monitoring, through one electrode with a microneedlearray. This work presents the: (1) device modeling and microneedle-array' fabrication method, (2) signal processing and biasing circuitry' design and simulation, (3) Analog Front End (AFE) for measured signals, and (4) Glucose sensor characterization. Design parameters and geometries are obtained by solving the capillarity model inside the microneedles and running optimization numeric methods. The AFE consists in a differential band pass filter that provides amplification, filtering, and noise rejection. This work presents clear technological innovation, for its miniaturization and integration of known biological signals' measurement methods in a portable Smart System, which points in the direction of Internet of Things' goals.
\end{abstract}

Keywords: Electrocardiogram, ECG, Glucose, Sensor, Microneedles, MEMS, Electrodes, Mobile Phones, Cellphones, Electronics, Biological Signals, Healthcare, Portable

\section{Introduction}

Many organs in human body, such as heart, brain, and muscles, produce measurable electrical signals [1] and their detection is necessary to collect clinical information that permits health diagnosis that many times prevent body failures. In order to monitor health conditions such as Diabetes, it is also necessary to evaluate enzyme's concentration in body fluid [2]. 
According to the World Health Organization, cardiovascular diseases are the main cause of death in the world and 422million people worldwide had diabetes in 2015, causing an average of 1.5 milion deaths per year, as in 2012 (http://www.who.int/diabetes/en/). Portable sensors that can measure biological signals related with those illnesses and prevent body failure, are desired to be integrated in our normal life. Further more, the healthcare' current and future trend is to give patients the ability to monitor their biological signals, making health diagnosis and at certain times preventing serious health conditions, during their normal day life, reducing significantly the hospital costs. Mobile phones are excellent candidates to be carriers of such portable sensors, due to their omnipresence in our daily lives and because general population keeps them near themselves, as much as possible, for communication purposes. This work intends to combine those facts proposing a MEMS system that integrates both sensing methods (ECG - Glucose) in one electrode, using microneedles.

One of the most important techniques to measure electrical signals in clinic and biomedical application is the ECG (electrocardiogram), which records the electrical activities of the heart over time [1][3][4][5][6][7]. The standard commercial ECG electrodes utilize an electrolytic gel to form conductive interface between skin and electrode. Using modern microsystem design techniques, it is possible to eliminate the use of gel by creating and using microneedles as electrodes [1][4][8]. At the same time, those microneedles can be used to extract interstitial fluid (ISF) to perform enzyme based diagnostic test, as shown in [9][10][11][12]. Due to careful microneedle array design the sensor is painless, because the length of microneedles is less than $500 \mu \mathrm{m}$ and so they don't reach the pain sensitive dermis' area, which will be explained in section 3.2.1. These concepts were applied in this device.

This work is divided in three main areas: microneedle fabrication, microfluidics for glucose sensor, and sensors' and AFE' electrical characterization. The microneedles for ECG electrodes and glucose electrodes share the same fabrication process. In this approach the microfluidic and electric models can be separated, because the ISF is static inside the channel during the glucose measurement. The link between both electrical sensors is the reference signal. A circuit for amplifying, eliminating noise and filtering is proposed for both systems, to connect them with a microcontroller.

The PhD study in which this work is being developed has the following research question and hypothesis: Research question: What would be a suitable way to do a low power system on chip (100s of $\mu \mathrm{W})$ that performs advanced computations for low frequency signals (below $10 \mathrm{kHz}$ ) as the brain does, as for example pattern recognition - namely Electrocardiogram (ECG) complete Arrhythmia detection, or fully implanted closed loop Brain Machine Interface (BMI) with brain signals' complex interpretation; without the use of a general purpose CPU? Hypothesis: One possible way is to develop a system on chip inspired in the brain that uses neurotechnology and neuromorphic circuits, creating an amplitude to time conversion with an Integrate and Fire circuit, which gives a stream of pulses. That system can then use pulse processing to do computations with the output pulses, for example with an automaton - without using conventional digital signal processing (DSP). If such kind of system is designed and prototyped then it may do these advanced computations with power consumption below $100 \mu \mathrm{W}$. Because its pulse timing 
structure only has information for relevant input amplitudes defined by the user, reducing the redundancy and lowering the hardware specifications.

The motivation of this work is presented next: With this power consumption, the system could be implanted in the Brain as a battery less standalone device to compensate for loss brain function, or could be used as a small ECG recording and arrhythmia detection device that is in contact with the patient chest. Currently these kinds of devices don't exist for ECG, or have very simple functions in the case of BMI, due to the limitation in computation power of such systems on chip.

\section{Contribution to Smart Systems}

This work presents clear technological innovation in a portable cellphone with smart sensors that measure body signals of the upmost importance, as for its miniaturization and integration of known biological signals' measurement methods in a portable Smart System, which points in the direction of Internet of Things' goals.

Moreover, the present work shows a clear contribution to Smart Systems as it paves the path of integrating healthcare and fitness solutions directly in mobile devices, that are one of the most used nodes to access Internet of things (IoT). In an IoT era, these are important developments to improve humans' daily life.

\section{Device Design}

The system was divided in four major modules to facilitate the design process: the microfluidic model that governs the microneedle fabrication, the glucose sensor, the ECG electrode, and the AFE for both physical measurements. Every module is independent from the others in terms of modeling, simulations and development predictions. The microfluidic model and the glucose sensor can be independent, because the method described in this document to monitor the glucose is for zero flow measurements. In the other hand, the ECG and the glucose sensor can be independent, because they will only share the reference electrode and the voltage in this electrode can be set as a design parameter for both modules.

\subsection{Capillarity Modeling}

Every microneedle that will be inserted inside the dermis of the human skin will experiment a capillarity drawn action. As explained in [9], [13], and [14], the ISF inside the dermis area of the skin will have a different pressure as compared with the atmosphere. Difference in pressures and the interactions of the fluid with the needle walls will create forces (van der Waals, dipole-dipole interaction, London dispersionfluctuating induced pole, and hydrogen bonds) that will contribute to changes on the surface tension of the fluid at the interface. Changes on the surface tension will generate the so-called capillarity drawn. Equation (1) shows the pressure difference in 
terms of liquid-gas surface tension $\left(\gamma_{\mathrm{lg}}\right)$ the contact angle between the liquid $(\theta)$, the volume and the solid $\left(\mathrm{A}_{\mathrm{sl}}\right)$ and liquid area $\left(\mathrm{A}_{\mathrm{lg}}\right)$.

$$
P=\gamma_{\mathrm{lg}}\left(\cos \theta\left(\frac{\mathrm{d} A_{\mathrm{sl}}}{\mathrm{d} V}\right)-\frac{\mathrm{d} A_{\mathrm{lg}}}{\mathrm{d} V}\right)
$$

Figure 5.4, page 96, of reference [14] presents the capillary rise in a vertically standing cylindrical microchannel. As shown in that figure, the liquid will flow inside the needle until it reaches the distance $\mathrm{h}$. A simplified model for $\mathrm{h}$ was demonstrated in [13] and can be expressed as:

$$
\mathrm{h}=\left(2 \cdot \gamma_{\mathrm{lg}} \cdot \cos \sigma(\theta)\right) /(\rho \cdot \mathrm{g} \cdot \mathrm{a}) .
$$

Where $\rho$ is the fluid density, $\mathrm{g}$ the gravity constant, and a the needle' radius.

This maximum drawn distance $h$ will be used later in this paper to optimize the design geometry.

\subsection{Glucose Sensor}

\subsubsection{Operating Principles}

The relationship between glucose concentrations in ISF and blood was studied in the past. In reference [15] the author compared the venous, capillary blood glucose and ISF glucose concentration in one subject during 3 days. A strong correlation was found which could lead to the useful method of measuring the glucose concentration from ISF instead of directly from blood. As the ISF can be extracted from the first layers of the skin, the measurement of glucose concentration from ISF can be painless. This is accomplished by making the microneedles for extraction of ISF smaller than $500 \mu \mathrm{m}$ long so they don't touch nerve cells when inserted in the skin. This is not possible in the extraction of blood that is always a process with some degree of pain. Thus, a small microneedle based glucose sensor can be designed. This is a major advantage of ISF glucose sensors as they can be painless, if designed properly. Comparing to usual commercially available blood glucose meters, for which the sample extraction is painful and can cause sensibility and irritation in the area of skin where the sample was taken, possibly making the user to have to switch the measurement location, between measurements.

Fig. 1 presents the glucose sensor' cross section view. The interstitial fluid (ISF) collected by the microneedle first flows over glucose oxidase (GOX), which is immobilized in the channel, Fig. 1. GOX will catalyze the glucose oxidation process to generate gluconic acid and hydrogen peroxide, equation (3). Then hydrogen peroxide when in contact with the working electrode will be decomposed into water and electrons, equation (4). Such electrons increase the ISF's electrical conductivity. A $0.7 \mathrm{~V}$ potential difference is added between the working electrode and the reference electrode that will induce a current. According to the whole reaction's equation the concentration of glucose in ISF is proportional to this current amplitude [10]. 


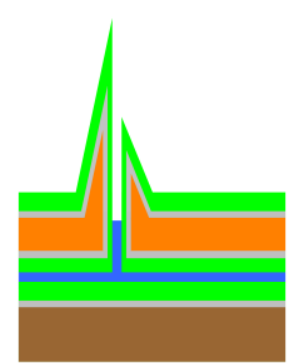

ECG Electrodes

P-Type Si (100) Group's Wafer

$\mathrm{Ag}(10 \mathrm{~nm})$

$\mathrm{AgCl}(200 \mathrm{~nm})$

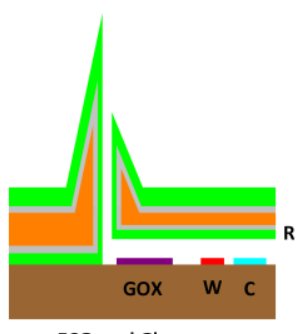

CG and Glucose electrodes

Immobilized Glucose Oxidase

Counter Electrode $(\mathrm{Pt})$

Working Electrode (Pt)

Conductive Adhesive

Fig. 1. Microneedle Cross section for the ECG electrodes (left) and ECG and glucose sensor electrode (right), W: working electrode, C: counter electrode, R: reference electrode, GOX: Enzyme.

$$
\begin{aligned}
\text { Glucose }+\mathrm{O}_{2} & \rightarrow \text { Gluconicacid }+\mathrm{H}_{2} \mathrm{O}_{2} . \\
\mathrm{H}_{2} \mathrm{O}_{2} & \rightarrow \mathrm{O}_{2}+2 \mathrm{H}^{+}+2 e
\end{aligned}
$$

\subsubsection{First-order Model}

The ISF inside of the channel can be treated as a linear resistor when the glucose concentration is small and a nonlinear one when the concentration is large. The lumped element model is simply a voltage source connected with a resistance in parallel. The governing equation of the reaction is Michaelis-Menten kinetics equation is:

$$
i=\frac{I_{\max }[S]}{K_{m}+[S]} .
$$

Where $i$ is current that is generated. $I_{\max }$ is the maximum current achieved by the system. $[S]$ is concentration of substrate. $K_{m}$ is Michaelis constant, which is the substrate concentration when current is half of $I_{\max }$.

The linearity between current and concentration depends on the assumption that the oxygen concentration in the ISF is enough. In reality, its concentration is limited. So when glucose concentration is more than $200 \mathrm{mg} / \mathrm{dl}$, it is nonlinear. The commercial glucose measurement devices based on this principle also present a nonlinear region above $200 \mathrm{mg} / \mathrm{dl}$.

From figure 3 of [16], that is based in the original data and curves of M. Daly, et al. [17], it can be seen that the fluctuation of blood sugar in normal humans during a day with three meals is less than $125 \mathrm{mg} / \mathrm{dl}$. The proposed glucose sensor in this paper 
will work properly, because the linear region of the sensor is larger than the ordinary human glucose concentration region: $3 \mathrm{mg} / \mathrm{dl}$ to $125 \mathrm{mg} / \mathrm{dl}$ [16], [17]. Moreover, when the blood sugar is at higher values, it is still possible to make a measurement by using a table stored in the micro controller memory.

\subsubsection{Glucose Sensor Device Performance}

The prospective dynamic range of glucose meter is: $0-180 \mathrm{mg} / \mathrm{dl}$, Fig. 2 . The resulting current is in the range 0 to $0.86 \mathrm{nA}$. Thus, the sensitivity is $0.0048 \mathrm{nA} * \mathrm{dl} / \mathrm{mg}$. This small current requires that the AFE has a high amplification gain, only possible with two or more amplifiers stages. Above a glucose concentration of approximately $180 \mathrm{mg} / \mathrm{dl}$, the device response is in the nonlinear region.

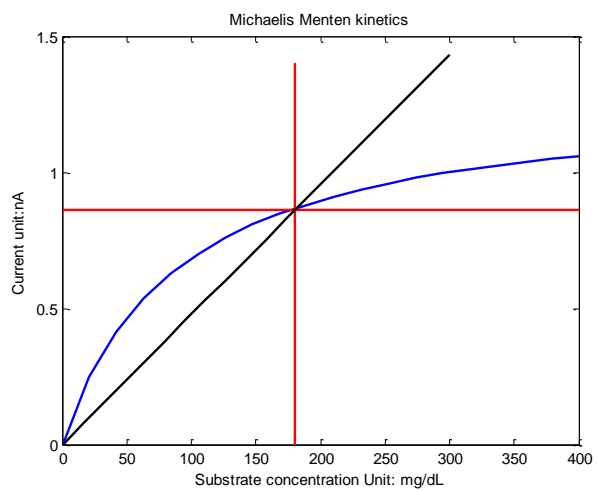

Fig. 2. The performance of glucose sensor, current versus substrate (glucose) concentration. The relation between glucose concentration and current is in blue. The perpendicular lines show the prospective dynamic range

\subsection{ECG Electrodes}

The designed finger ECG electrodes consist in dry electrodes that have a 3D shape. The electrodes are deposited on top of an array of micro needles to decrease the overall electrode' impedance. Each ECG electrode consists in a 2-wafer structure. The top wafer is micro fabricated to have an array of hollow micro needles. After this microfabrication, the electrodes' metal $(\mathrm{AgCl}+\mathrm{Ag})$ is sputtered in all the sides of the wafer and due to the microneedles geometry it is ensured that there is an electric contact between both sides of the wafer, through the micro needles' holes, Fig. 1. The bottom wafer for the ECG electrode has a metal line that makes a contact between the top wafer and the electronic circuitry. Both wafers are joined together by an $\mathrm{Ag}$ conductive glue, in the case of the 2 ECG electrodes, without the glucose sensor.

The extraction of ECG signal from the finger is a novel method. In [3] is presented a proof a principle of finger ECG, using commercial planar dry electrodes and in [5] is presented an heart beat rate monitor system that uses planar dry electrodes for the 
fingers. The micro needle based electrode for ECG is also a novel approach. Reference [8] shows the microneedle device with a chamber, in the back of the microneedle structure, containing a conductive gel, to improve the conductance of these type of electrodes. It has been shown that dry microneedle electrodes are capable of acquiring signals from ECG, electromyography (EMG) and even electro encephalography (EEG), signals that are lower voltage signals, than ECG and EMG, and so require a very low impedance electrode, [1] and [4]. These microneedles's electrodes were designed to record ECG in the human chest. As an ECG measurement device has never been integrated directly in a cell phone.

The first order model for the ECG measurement and the lumped element model for the skin and electrode is presented in [1] and [18]. To do an ECG measurement it is required to use two active electrodes, in two fingers, or one finger and hand, and a third reference electrode in the other hand, or in another finger, or in a different zone.

\subsubsection{ECG Device Performance}

An estimate of the ECG electrodes' device performance was made. The electrode area is the area of the whole device, without electronic circuitry, as the electrode metal layer is deposited over all the microneedles in the array: electrode area $=3.8 \mathrm{~mm} * 2.5 \mathrm{~mm}$ with a AgCL layer, $25 \mathrm{~nm}$ thick.

The maximum input signal range is $-1 \mathrm{mV}$ to $1 \mathrm{mV}$. The ECG electronic circuitry performs an amplification of 500 times, it has to have a linear response from $0.05 \mathrm{~Hz}$ until $500 \mathrm{~Hz}$, that is half of the sampling frequency so it can measure accurately each one of the peaks that make the ECG QRSPT complex, and measure a minimum detectable signal of $5 \mu \mathrm{V}$. These and other specifications are presented in the next tables:

Table 1. Overall circuit' specifications.

\begin{tabular}{ll}
\hline Specifications & Value \\
\hline Supply voltage Vss & $3.6 \mathrm{~V}$ \\
Imax (source) [7] & $40 \mathrm{~mA}$ \\
Power & $144 \mathrm{~mW}$ \\
\hline
\end{tabular}

Table 2. ECG AFE Specifications.

\begin{tabular}{ll}
\hline Specifications & Value \\
\hline Vbias [1] maximum & $1.65 \mathrm{~V}$ \\
$\begin{array}{l}\text { Input } \\
\text { signal range [5] }\end{array}$ & \\
MDS [6] & $5 \mu \mathrm{mV}$ \\
Micro needles height & $20 \mu \mathrm{m}$ \\
[2] & \\
Sampling frequency & $1 \mathrm{kHz}$ \\
(fs) [3] & \\
\hline
\end{tabular}


To accomplish these circuit requirements it is important that the circuit has low noise. More details about the electronic circuits are given in section 5 .

\subsection{Design Optimization}

In order to obtain the geometry values to generate the proper mask for the fabrication process it is necessary to analyze the behavior of the microfluidic module and its interaction with the human skin. Every microneedle will experiment the capillarity drawn for the ISF. It is necessary to design the system with the proper length so the ISF can fill the needle and the micro channel covering the electrodes. This will be the most important parameter to be considering for the mask design. Considering this parameter an optimization problem can be written in order to maximize the fluid volume inside the needle (cylinder volume) and the channel (rectangular channel). Fig. 3 shows the distances for the fluidic module.

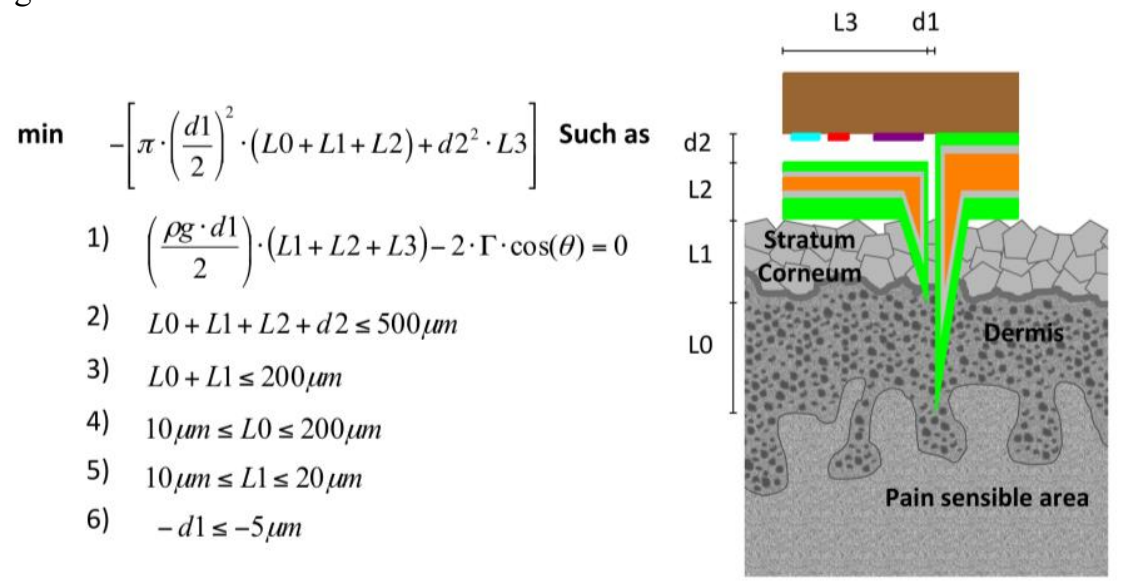

Fig. 3. Left: . Standard format for the optimization problem. Objective Function, constraints (from 1 to 3 ) and boundaries (4 to 6). Right: Microneedle inserted in human skin. Geometry variables used in the optimization process. Main dimension constrains related with skin interaction are shown.

The optimization problem can be written in the standard format as in Fig.3. Constrains for this problem include: 1) the capillarity equation shown in (2) with the ISF $\rho=1060 \mathrm{~kg} / \mathrm{m}^{3}$ [18], the ISF surface tension $\Gamma=60 \mathrm{~mJ} / \mathrm{m}^{2}$ [19], and the contact angle $\theta=110^{\circ}$ [20] between ISF and needle walls. 2) Represent the wafer thickness. 3) Is the maximum needle' dimension in order to avoid reaching dermis' pain sensitive area according to [6]. 4) is the dermis typical thickness for human skin. This area contains the ISF. 5) is the stratum corneum thickness for human skin. And 6) is a fabrication limitation by the $20 \mathrm{~mm}$ head of the Heidelberg laser stepper available in the University of Florida's Nanoscale Research Facility (NRF) clean room that will be used in this fabrication process. The Matlab ${ }^{\circledR}$ optimization results obtained, after rounding the numbers to fit the fabrication requirements on the available clean room, are: microneedle chamber length $\mathrm{L} 3=170 \mu \mathrm{m}$ and microneedle diameter $\mathrm{d} 1=20 \mu \mathrm{m}$. 


\section{Device Fabrication}

Device fabrication is summarized next:

A. Backside deep reactive ion etching (DRIE) Bosh process $(360 \mu \mathrm{m})$ using Aluminum (Phosphoric - Acetic - Nitric (PAN) etched) and Photoresist as mask.

B. Backside reactive ion etching (RIE) process $(15 \mu \mathrm{m})$ using Aluminum (PAN etched).

C. Front side DRIE Bosh process $(170 \mu \mathrm{m})$ using thick Photoresist as mask.

D. Sharpening etching (HNA solution)

E. Backside metal deposition (Ag) using sputtering and electrochemical deposition (TSV).

F. Front side metal deposition (Ag) using sputtering and electrochemical deposition (through-silicon via (TSV)) [9].

G. $\mathrm{FeCl} 3$ chemical oxidation to generate the $\mathrm{AgCl}$ and finalize the $\mathrm{Ag} / \mathrm{AgCl}$ deposition of the TSV on both sides.

H. Metal deposition for electrodes on the Group's Wafer. For electrodes in the glucose sensor.

I. Glucose immobilization.

J. Silicon/Silicon anodic bounding for glucose sensor and conductive adhesive bounding for ECG electrodes.

\section{Integration and Packaging}

\subsection{Mounting and Packaging}

The device is integrated in a System in Package (SiP) that is inside the cellphone. The system is controlled by the ultra-low power microcontroller MSP430FR5739, from Texas Instruments [21], [22]. The biosensor device, as was previously explained, consists in three different electrode structures: two dry ECG sensors and a third wet sensor for ECG and Glucose detection. One of the dry ECG electrodes (active electrode) is integrated in the system in package with the signal processing electronic circuitry. The other dry electrode (reference electrode) is also inside the cellphone, but is connected with a cable to the SiP. The third sensor includes an active ECG electrode and Glucose sensor, this last sensor is disposable and a platform similar to a SD card platform is used to change this disposable sensor. A cable connects this sensor to the electronic circuitry in the SiP. The disposition of these three sensors is shown in Fig. 4. 


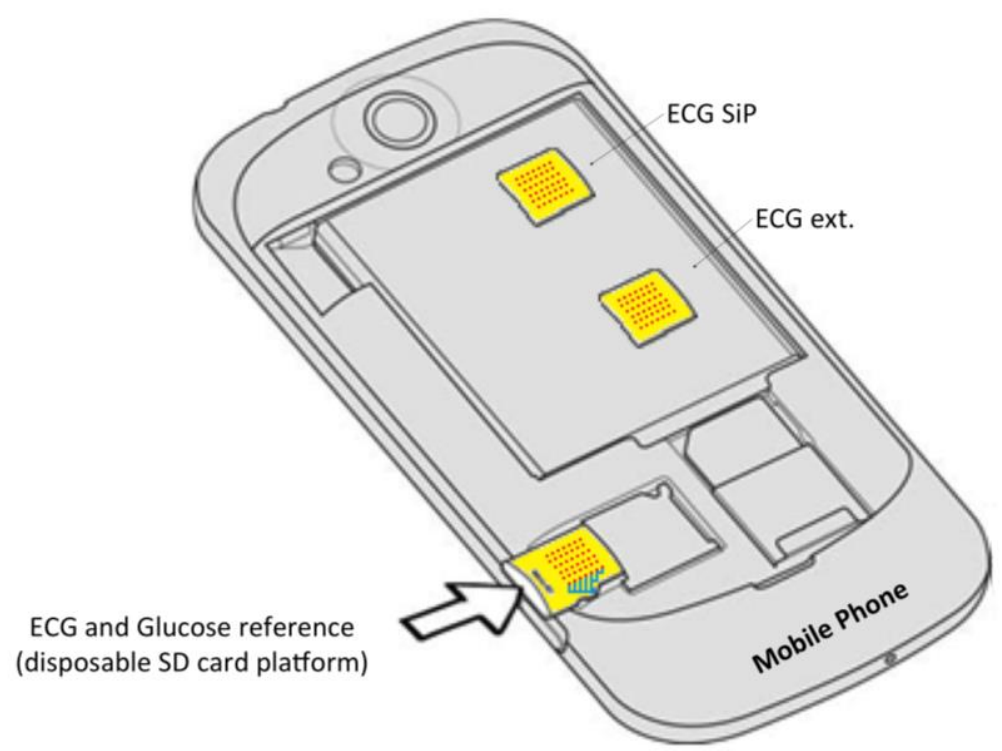

Fig. 4. Schematic example of Sensors and SiP disposition in the mobile phone

As was referred before both ECG dry electrodes and the glucose and ECG sensor consist in two Si wafers. The bottom wafer for the ECG electrode has a metal line that makes a contact between the top wafer and the bottom wafer and then connects to the electronic circuitry. Both wafers are joined together by an Ag conductive glue, in the case of the 2 ECG electrodes, without the glucose sensor. The contact in the bottom wafer is wire bonded to a wafer with the electronic circuitry and the microcontroller.

In the glucose and ECG sensor, the two wafers are joined by anodic bonding and the bottom wafer contains metal lines that are connect to a cable connector by wire bonding, this system is all inside the SD card platform.

\subsection{Signal Processing Electronic Circuitry}

The two active ECG electrodes are connected to the electronic signal processing circuitry similar to the one shown in figure 2 of [23], these are connected to a differential amplifier that is connected to a high pass filter amplifier that eliminates the DC voltage and then the signal goes through a high gain low pass filter amplifier that connects to and ADC channel of the microcontroller. The overall total gain of this amplifier is 500. Spice simulations were made using BSIM 3 models for MOSIS $350 \mathrm{~nm}$ technology. A band pass filter with two amplifier stages and a capacitor feedback configuration was designed, Fig. 5. 


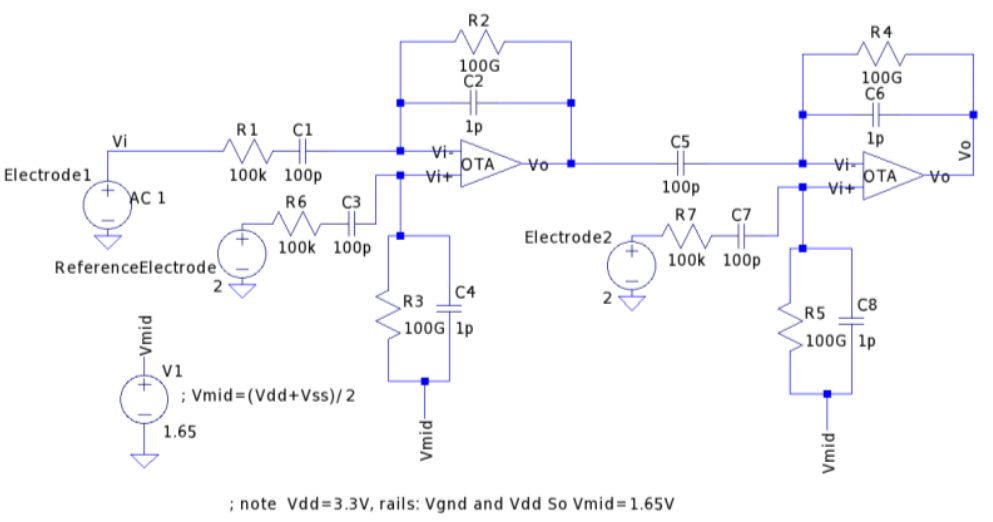

Fig. 5. Single ended Band Pass filter with Capacitor feedback for ECG AFE.

This circuit is a band pass filter. The negative feedback loop defined by R2 and C2 gives the high pass frequency of the circuit and R1 C1 plus the low pass filter behavior of the amplifier (any non ideal amplifier due to the devices, transistors and the way they behave in the amplifier stages has intrinsically a Gain roll of frequency that gives a low pass filter behavior) define the low pass frequency. The feedback gain is defined by $\mathrm{Cin} / \mathrm{Cf}=\mathrm{C} 1 / \mathrm{C} 2$, being $\mathrm{Cin}$ the input capacitance and $\mathrm{Cf}$ the feedback capacitance [24] and [25], and in this example it is 100, note for just one amplifier stage. This structure is very common in bio amplifiers and provides good results [25]. R2 and R4 are pseudoresistors to achieve a high resistance value. Each amplifier stage consists in a single ended dual input, PMOS and NMOS input, current mirror Output Transconductance Amplifier (OTA) presented in Fig. 6.

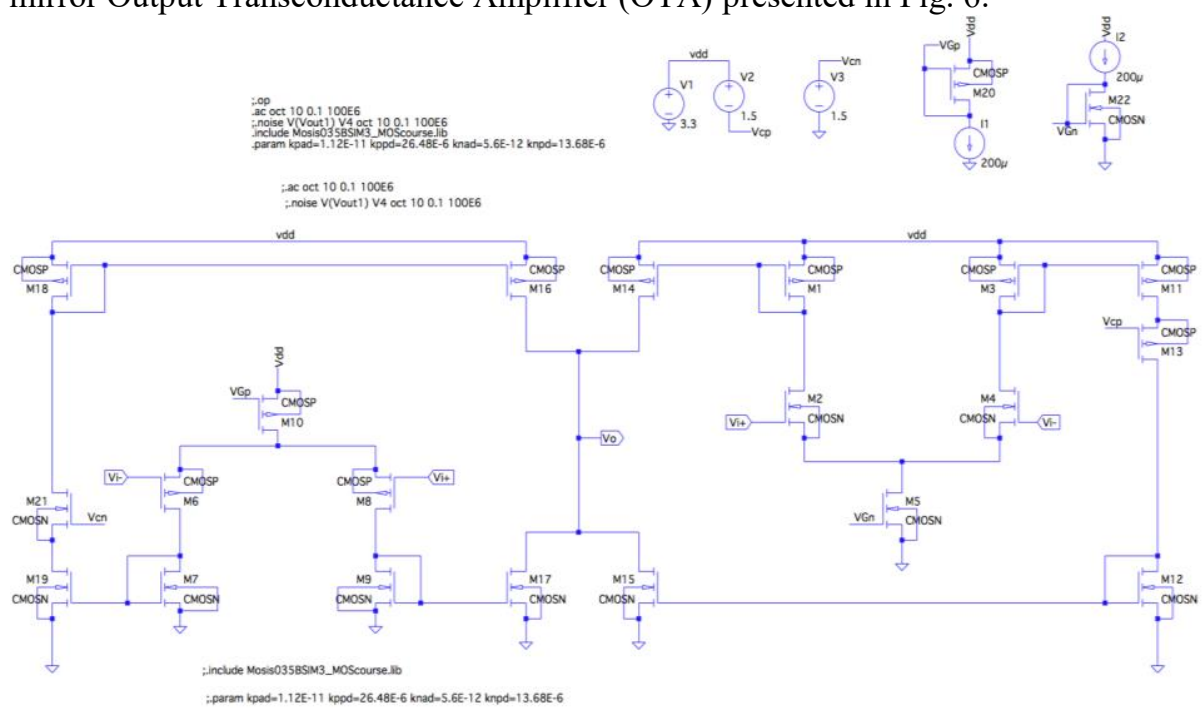

Fig. 6. Single ended dual input, PMOS and NMOS input, current mirror OTA. 
The glucose sensors are also connected to electronic circuitry. Fig. 6 As the glucose sensor has 100 microneedles the signals from each electrode are connected in parallel (working electrodes' signals are connected in parallel, and reference electrodes' signals are connected in parallel). This way the measured current is approximately 100 times the current of a single microneedle, so in the order of $100 \mathrm{nA}$. This is considering that the reaction in all microneedles is approximately at same stage and in the maximum current reaction point, of $1 \mathrm{nA}$ per microneedle. The overall signal of the working electrodes and the signal from the reference electrode are then connected to the signal processing circuitry. The current from the working electrodes is converted to a voltage in a transimpedance amplifier and then it is connected to a differential amplifier together with the signal from the reference electrodes. The signal is then amplified in a high gain low pass filter amplifier and connected to another channel of the microcontrollers ADC. The overall gain of this amplification circuit should be in the order of $90 \mathrm{~dB}$, considering the total gain of the three amplification blocks.

The interface circuitry has a major influence in the system performance, because the sensors are measuring very small signals, the minimum detectable signal (MDS) of ECG is $5 \mu \mathrm{V}$ and the current detected in the glucose sensor array is of the order of $100 \mathrm{nA}$. In this sense, noise is one of the main issues. To minimize noise: the system must have well designed fully differential amplifiers, the gain of the first amplifier stage should be maximized for each amplifier comparatively with the other stages. Also there should be a good electromagnetic insulation from the cellphone circuitry, especially from RF.

\section{Results}

A numeric optimization for the microfluidic parameters was done and the obtained results are: microneedle chamber length $\mathrm{L} 3=170 \mu \mathrm{m}$ and microneedle diameter $\mathrm{d} 1=20 \mu \mathrm{m}$.

The layout of the fabrication masks for the glucose sensor device with the microneedle array for all the material layers and respective fabrication steps was made using the Ledit ${ }^{\circledR}$ software. Considering the fabrication process and as result of

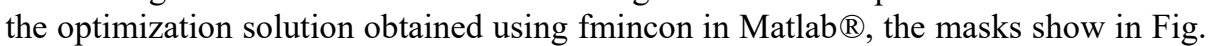
7 were created using Ledit $\AA$ software for layout design. 


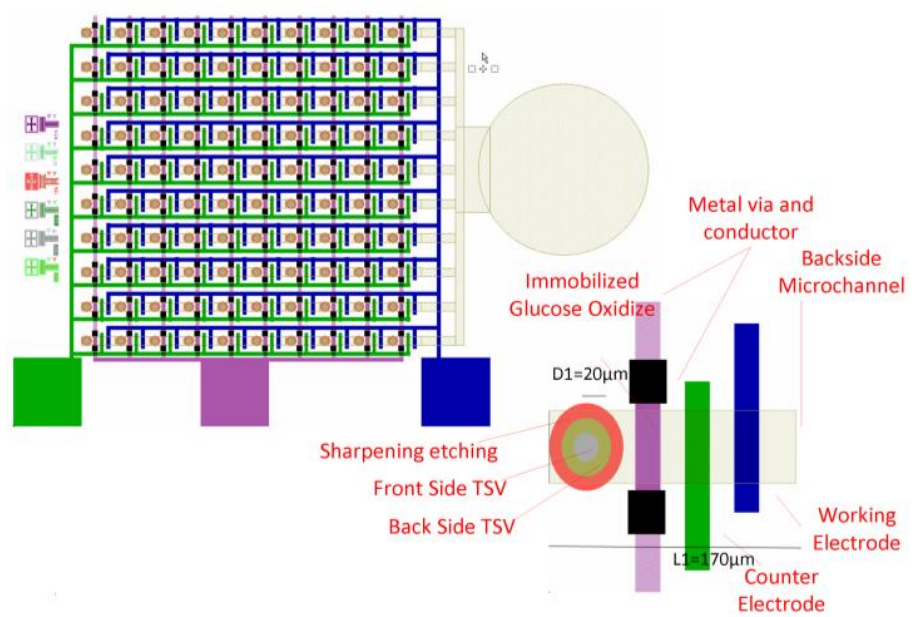

Fig. 7. Array of 10 needles by 10 needles design layout, made with Ledit ${ }^{\circledR}$ software, and closeup of a single microneedle' layout with its own glucose sensor electrodes.

Fig. 7 presents the final layout of the sensors array with the array of 10 needles by 10 needles, the electrical pads for the glucose sensing and the interconnection channels with a general outlet for generating the pressure difference. Alignment marks for the mask (global alignment, vernier and photoresists development) were also included in this layout. The final system (glucose sensor) area is $3.8 \mathrm{~mm} \mathrm{x}$ $2.5 \mathrm{~mm}$.

The AFE simulations results met the design specifications. Fig. 8 presents the transient analysis with a real ECG noisy signal with typical signal wander due to movement and variation in the electrodes' contact with skin. And Fig. 9 presents the AFE Bode plot from the AC analysis. Table 3 presents the AFE simulation results.

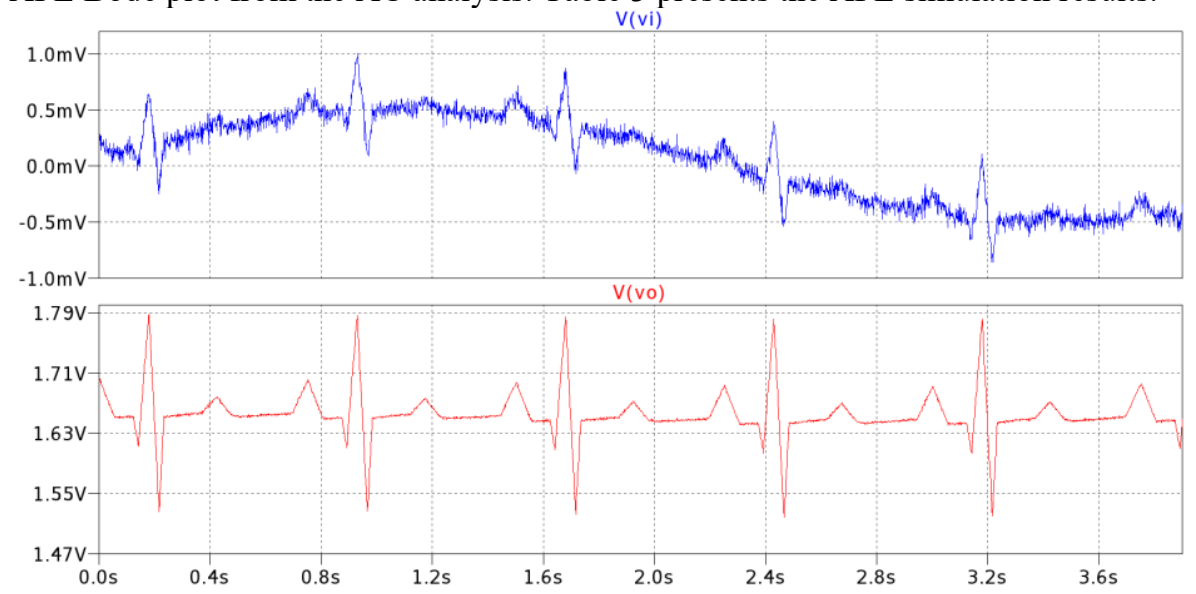

Fig. 8. Transient AFE Simulation Results - Top: input signal ECG in blue with noise and signal wander, Bottom: AFE signal output in red. 


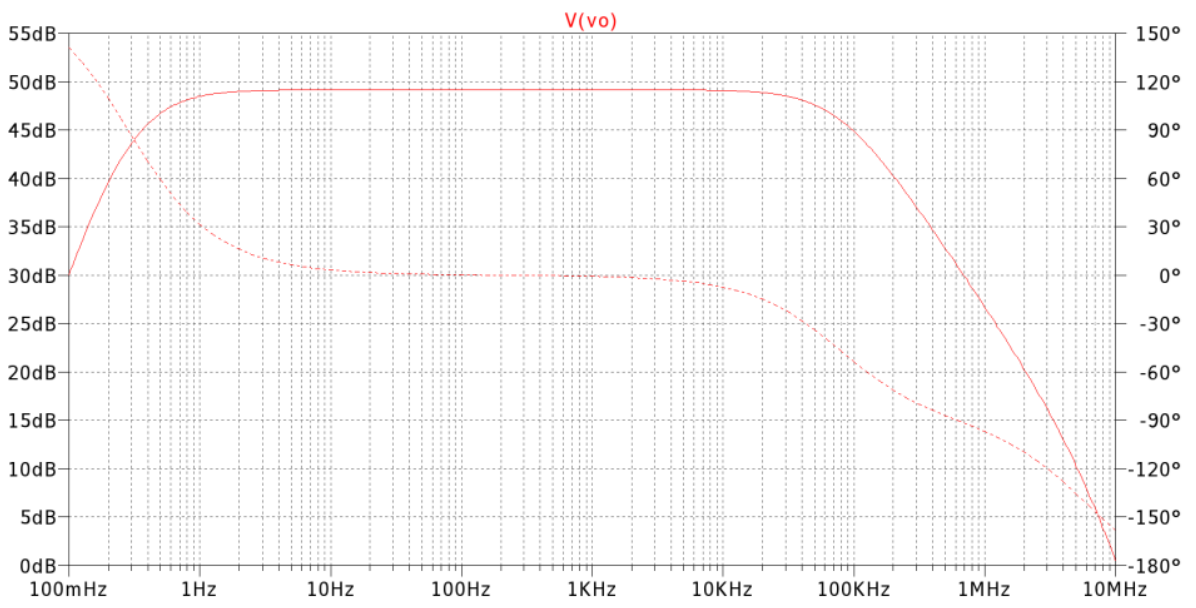

Fig. 9. AFE band pass filter Bode plot for the two stages together.

Table 3. ECG AFE Simulated Specifications.

\begin{tabular}{ll}
\hline Specifications & Value \\
\hline Supply Voltage Vdd & $3.3 \mathrm{~V}$ \\
Imax (source) & $2 \mathrm{~mA}$ \\
Power maximum & $6.6 \mathrm{~mW}$ \\
$\begin{array}{l}\text { Input } \\
\text { signal range }\end{array}$ & \\
$\begin{array}{l}\text { Vbias and Vcm } \\
\text { (common mode) }\end{array}$ & \\
$\begin{array}{l}\text { Gain } \\
\text { 3dB Bandwidth }\end{array}$ & $49 \mathrm{~dB}(282 \mathrm{x})$ \\
$\begin{array}{l}\text { Common Mode } \\
\text { Rejection } \quad 52 \mathrm{~dB}(391 \mathrm{x}) \\
\text { (CMRR) Ratio }\end{array}$ \\
\hline
\end{tabular}

\section{Conclusion}

A novel minimal invasive micro needle based ECG and Glucose sensor was designed. The whole sensor can be integrated on smart phone using a system in package approach. The extraction of ECG signal from the finger with a microneedle array is a novel approach. The ECG AFE has $2 \mathrm{~mW}$ power consumption that can be further reduced, by reducing the band pass filter bandwidth. Considering the design constraints and fabrication tools, a Matlab ${ }^{\circledR}$ design optimization was made for the microneedle array obtaining a microneedle chamber length L3=170um and a microneedle diameter $\mathrm{d} 1=20 \mathrm{um}$. The fabrication steps and respective micro and nano fabrication masks were developed. The final glucose sensor area, that consists in in a array of 10 by 10 microfabricated microneedles is $3.8 \mathrm{~mm} \times 2.5 \mathrm{~mm}$. The sensor has a 
$0.0048 \mathrm{nA} * \mathrm{dl} / \mathrm{mg}$ sensitivity for glucose sensing and an estimated dynamic range of $0-180 \mathrm{mg} / \mathrm{dl}$ and so it is suited to be used for daily ISF sugar monitoring. The ECG AFE was designed and simulated complying with all the design specifications. It has a Gain of $49 \mathrm{~dB}$ and a good CMRR of $52 \mathrm{~dB}$.

Some future challenges are:

1. How to expand the linear range of glucose sensor. (oxygen integrated). A possible way is to integrate some spare oxygen in an air bag, which is soluble to ISF. Making the saturation region smaller.

2. The cost per sensor is high due to electrode material and fabrication process. Ways to minimize cost should be studied and developed.

3. Also a method to avoid microneedle loss because of breakage, or use the option of making all sensors disposable should be considered and studied

4. The pseudo resistors used in the ECG AFE are not a definitive solution, because their value is not accurate enough, it is quite sensitive to process, voltage and temperature variations (PVT). It changes with process variation from chip to chip, with the slight differences in feature size and for the same reason the simulated values are different than the actual values on chip, making the band filter $3 \mathrm{~dB}$ corner frequency slightly different than simulated and expected. This is specially an issue for the low pass frequency that should to be in the tight range of $0.05 \mathrm{~Hz}$ to $0.5 \mathrm{~Hz}$.

This work shows a clear contribution to Smart Systems as it paves the path of integrating healthcare and fitness solutions directly in mobile devices, that are one of the most used nodes to access Internet of things (IoT). In an IoT era, these are important developments to improve humans' daily life.

\section{References}

1. O’Mahony C, Pini F, Blake A, Webster C, O’Brien J, McCarthy KG (2012) Microneedlebased electrodes with integrated through-silicon via for biopotential recording. Sensors Actuators A Phys 186:130-136. doi: 10.1016/j.sna.2012.04.037

2. Newman JD, Turner APF (2005) Home blood glucose biosensors: a commercial perspective. Biosens Bioelectron 20:2435-2453. doi: 10.1016/j.bios.2004.11.012

3. Lourenço A, Silva H, Fred A (2011) Unveiling the biometric potential of finger-based ECG signals. Comput Intell Neurosci 2011:1-8. doi: 10.1155/2011/720971

4. Forvi E, Bedoni M, Carabalona R, Soncini M, Mazzoleni P, Rizzo F, O’Mahony C, Morasso C, Cassarà DG, Gramatica F (2012) Preliminary technological assessment of microneedles-based dry electrodes for biopotential monitoring in clinical examinations. Sensors Actuators A Phys 180:177-186. doi: 10.1016/j.sna.2012.04.019

5. Raju M (2007) Heart-Rate and EKG Monitor Using the MSP430FG439.

6. Sarpeshkar R (2010) Ultra Low Power Bioelectronics Fundamentals, Biomedical Applications, and Bio-Inspired http://dx.doi.org/10.1017/CBO9780511841446

7. Fulford-Jones TRF, Wei G-Y, Welsh M (2004) A portable, low-power, wireless two-lead EKG system. In: Conf. Proc. 26th Annu. Int. Conf. IEEE EMBS. pp 2141-2144

8. Yu LM, Tay FEH, Guo DG, Xu L, Nyan MN, Chong FW, Yap KL, Xu B (2008) A MEMS-based bioelectrode for ECG measurement. In: 2008 IEEE Sensors. Ieee, pp 10681071 
9. Mukerjee EV, Collins SD, Isseroff RR, Smith RL (2004) Microneedle array for transdermal biological fluid extraction and in situ analysis. Sensors Actuators A Phys 114:267-275. doi: 10.1016/j.sna.2003.11.008

10. Wang PM, Cornwell M, Prausnitz MR (2005) Minimally invasive extraction of dermal interstitial fluid for glucose monitoring using microneedles. Diabetes Technol Ther 7:131141. doi: 10.1089/dia.2005.7.131

11. Zimmermann S, Fienbork D, Stoeber B, Flounders AW, Liepmann D (2003) A microneedle-based glucose monitor: fabricated on a wafer-level using in-device enzime inmobilization. In: 12th Int. Conf. Solid State Sensors, Actuators Microsystems. pp 99-102

12. Zhao Y, Li S, Davidson A, Yang B, Wang Q, Lin Q (2007) A MEMS viscometric sensor for continuous glucose monitoring. J Micromechanics Microengineering 17:2528-2537. doi: $10.1088 / 0960-1317 / 17 / 12 / 020$

13. H.Bruss (2008) Theoretical Microfluidics. Oxford University Press

14. Bruus H (2006) Theoretical microfluidics, Third Fall. Lect Notes Fall 2006. doi: 10.1111/j.1574-6968.2009.01808.x

15. Thennadil SN, Rennert JL, Wenzel BJ, Hazen KH, Ruchti TL, Block MB (2001) Comparison of Glucose Concentration in Interstitial Fluid, and Capillary and Venous Blood During Rapid Changes in Blood Glucose Levels. 357-365. doi: $10.1089 / 15209150152607132$

16. Suckale J, Solimena M (2008) Pancreas islets in metabolic signaling - focus on the Betacell. 7156-7171.

17. Daly ME, Vale C, Walker M, Littlefield A, George K, Alberti MM, Mathers JC (1998) Acute effects on insulin sensitivity and diurnal metabolic profiles of a high-sucrose compared with a high-starch diet. Am J Clin Nutr 67:1186-1196.

18. Dorgan SJ, Reilly RB (1999) A Model for Human Skin Impedance During Surface Functional Neuromuscular Stimulation. 7:341-348.

19. Cutnell J, Johnson K (1998) Physics, Fourth. Wiley

20. ASM International (2009) Materials and Coatings for Medical Devices: Cardiovascular. ASM International

21. Texas Instruments (2013) Texas Instruments MSP430FR5739 Mixed Signal Microcontroller. http://www.ti.com/product/msp430fr5739.

22. Texas Instruments (2013) MSP430FR573x MSP430FR572x Mixed Signal Microcontroller datasheet.

23. Msp MR (2007) Heart-Rate and EKG Monitor Using the MSP430FG439. 1-12.

24. Xiao Z (2013) Low Power Circuits and Systems for Brain Machine Interface. University of Florida

25. Wattanapanitch W, Fee M, Sarpeshkar R (2007) An Energy-Efficient Micropower Neural Recording Amplifier. IEEE Trans Biomed Circuits Syst 1:136-147. doi: 10.1109/TBCAS.2007.907868 